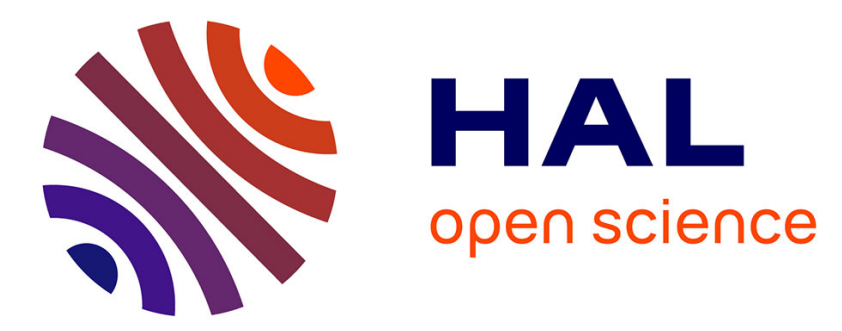

\title{
Silicon-on-Insulator RF Filter Based on Photonic Crystal Functions for Channel Equalization
}

Mathilde Gay, Laurent Bramerie, Sy Dat Le, Luiz Anet Neto, Jean-Claude Simon, Christophe Peucheret, Zheng Han, Xavier Checoury, Gregory Moille, Jerome Bourderionnet, et al.

\section{To cite this version:}

Mathilde Gay, Laurent Bramerie, Sy Dat Le, Luiz Anet Neto, Jean-Claude Simon, et al.. Silicon-onInsulator RF Filter Based on Photonic Crystal Functions for Channel Equalization. IEEE Photonics Technology Letters, 2016, 28 (23), pp.2756 - 2759. 10.1109/LPT.2016.2616162 . hal-01393206

\section{HAL Id: hal-01393206 https://hal.science/hal-01393206}

Submitted on 7 Nov 2016

HAL is a multi-disciplinary open access archive for the deposit and dissemination of scientific research documents, whether they are published or not. The documents may come from teaching and research institutions in France or abroad, or from public or private research centers.
L'archive ouverte pluridisciplinaire HAL, est destinée au dépôt et à la diffusion de documents scientifiques de niveau recherche, publiés ou non, émanant des établissements d'enseignement et de recherche français ou étrangers, des laboratoires publics ou privés. 


\title{
Silicon-on-insulator RF Filter based on Photonic Crystal Functions for Channel Equalization
}

\author{
Mathilde Gay, Laurent Bramerie, Luiz Anet Neto, Sy Dat Le, Jean-Claude Simon, \\ Christophe Peucheret, Zheng Han, Xavier Checoury, Grégory Moille, Jérôme Bourderionnet, \\ Alfredo De Rossi, and Sylvain Combrié
}

\begin{abstract}
A compact silicon-on-insulator 2-tap interferometer is demonstrated as a channel equalizer. The radiofrequency filter is reconfigurable thanks to thermally-controlled photonic crystal couplers and delay lines. The channel fading of a dispersive fiber link supporting a directly modulated telecommunication signal is successfully compensated for using the interferometer, leading to eye diagram opening and the possibility to recover the bit-errorrate of a reference signal with less than 1-dB power penalty.
\end{abstract}

Index Terms-Channel equalization, microwave optical filter, photonic crystals, photonic integrated circuits.

\section{INTRODUCTION}

$\mathrm{M}$ ICROWAVE photonic filters are an emerging echnology for digital, radio, and space communications as well as radars. Processing signals in the optical domain can provide spectral agility, broadband operation and dynamical reconfigurability [1]. A popular design for radio frequency (RF) photonic filters is based on discrete-time signal processing architectures. It consists in combining delayed replicas of the signal with suitably chosen weights. The optical phase stability of the signal contributions propagating in each arm of such a design is generally highly dependent on environmental parameters. This results in the so-called incoherent regime, where phase shifts implemented along the taps have no effect on the overall transfer function of the filter. The resulting power summation restricts such tap filters to have positive coefficients [2]. The related transfer function consequently exhibits a resonance at zero frequency, which is not suited for RF-bandpass filtering. Some solutions have been proposed in the literature to provide a $\pi$ phase shift between taps in the incoherent regime, thus allowing negative coefficients to be synthesized, notably using cross-gain modulation in semiconductor optical amplifiers (SOAs) [3] or using phase inversion in electro-optic modulators [4]. Incoherent regime filters with complex coefficients can also be realized by

Manuscript received March $1^{\text {st }}, 2016$. This work was supported in part by the French National Research Agency (ANR) under the SYMPHONIE (ANR2012-NANO-0011-01) project and by the RENATECH network.

M. Gay, L. Bramerie, S. D. Le, J. C. Simon, C. Peucheret are with the Foton Laboratory, CNRS, University of Rennes 1, Enssat, Lannion, France (e-mail: mathilde.gay@enssat.fr).

L. Anet Neto, was with Foton, Lannion, France. He is now with Orange Labs, Lannion, France. exploiting stimulated Brillouin scattering, resulting in tunable phase shifts [5].

The microwave photonic approach has further gained in interest with the recent development of manufacturing platforms for photonic integrated circuits (PICs) allowing the monolithic integration of a broad range of optical and RF functions on a very small footprint. Thanks to the inherent stability of monolithically integrated structures, these platforms make it possible to implement novel filter concepts operating in the so-called coherent regime, where the optical phase shifts induced in the taps play a predominant role in the overall response of the filter. As a consequence, filters with negative and complex coefficients can be implemented. RF-filters employing SOAs and phase modulators in an interferometric structure were notably demonstrated in III-V structures [6]. Another demonstration, using silicon-on-insulator (SOI) technology, involved a fixed delay-line in an interferometer whose taps weights were tunable [7].

Highly dispersive photonic crystal $(\mathrm{PhC})$ waveguides have been introduced recently to demonstrate integrable, compact and power-efficient microwave photonics devices, specifically, a microwave filter based on a $\mathrm{PhC}$ true-time delay-line [8]. That device was based on the incoherent summation of four channels, with the inherent limitations mentioned above.

In this paper, we demonstrate an SOI RF-filter tunable both in frequency and extinction, by exploiting thermally-controlled ultra-compact $\mathrm{PhC}$ coupler and delay line. The use of $\mathrm{PhC}$ technology allows footprint reduction (the coupler footprint is less than $15 \times 15 \mu \mathrm{m}^{2}$, including rib-access waveguides and integrated heaters; the delay line section length is $500 \mu \mathrm{m}$ allowing up to 70 ps delay), reduced power consumption (only a few $\mathrm{mW}$ are necessary to induce a $\pi$ phase shift of the filter) and improved heaters dynamics (small thermal capacitance leads to a time response in the $\mu$ s scale). The 2-tap filter is demonstrated for the first time for channel equalization in a $100-\mathrm{km}$ long link with intensity modulated telecommunication signals.

$\mathrm{X}$. Checoury is with the Institut d'Electronique Fondamentale (IEF), Univ. Paris-Sud, CNRS, Univ. Paris-Saclay, Orsay, France (e-mail: xavier.checoury@ief.u-psud.fr).

Z. Han was with IEF. He is now at MirSense, Palaiseau, France.

J. Bourderionnet, A. De Rossi, S. Combrié are with Thales Research and Technology (TRT), Palaiseau, France (sylvain.combrie@ thalesgroup.com).

G. Moille was with TRT, he is now with IEF, Orsay, Paris. 


\section{DEVICE DESCRIPTION}

The core of the filter is an unbalanced Mach-Zehnder interferometer implemented on a 220-nm-thick SOI platform, as depicted in Fig. 1(a). The long arm consists of a 1-cm long shallow (800-nm wide, $70-\mathrm{nm}$ high) ridge waveguide coiled into a double spiral, generating a fixed time delay, and a $500-\mu \mathrm{m}-$ long tunable delay-line based on a dispersionengineered $\mathrm{PhC}$ waveguide (Fig. 1(d)). The lattice period $a$ is $409 \mathrm{~nm}$, whereas the radius of the holes is $0.26 a$. The width of the waveguide is reduced to $0.9 \sqrt{ } 3 a$ bringing closer the blocs of $\mathrm{PhC}$. The first row of holes is shifted in opposite directions along the waveguide axis by $\mathrm{s}=0.12 a$. The third row of airholes was shifted by a distance $\mathrm{t}=0.1 a$ following [9]. These parameters result in a highly dispersive waveguide with almost linearly increasing group index with the wavelength. The PhC delay line is connected to ridge waveguides (460-nm wide and 220 -nm high) that are then adiabatically converted to shallow waveguides through inverted tapers (see Fig. 1(e)).
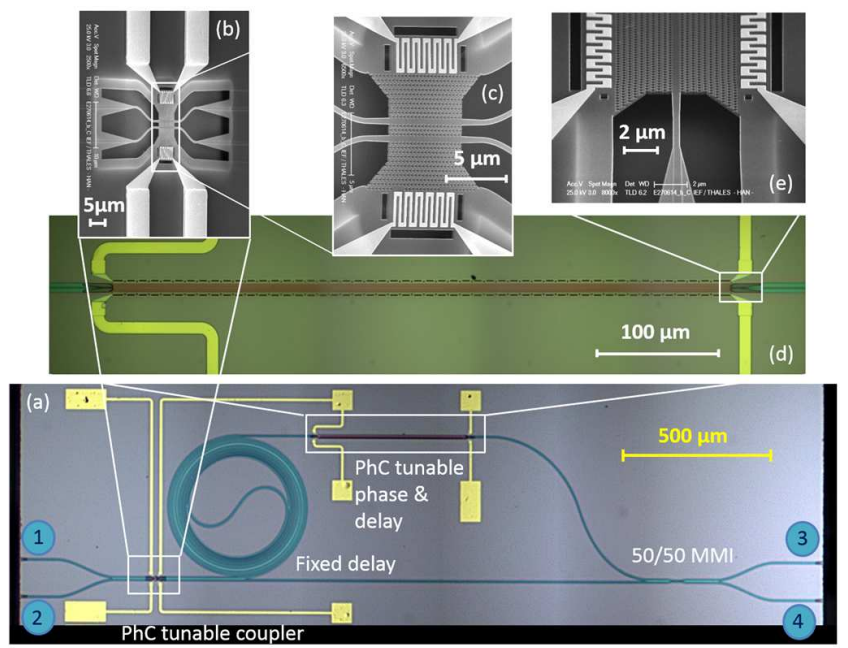

Fig. 1. (a) Picture of the device. (b), (c) SEM images of the directional coupler. (d) Picture of the PhC delay line. (e) SEM image of the PhC delay line extremity.

The short arm is a $1.7-\mathrm{mm}$-long shallow ridge waveguide. The path difference is $137 \mathrm{ps}$, corresponding to a free spectral range (FSR) of $7.2 \mathrm{GHz}$. The input power is split with a ratio that is controlled using a 8.3- $\mu \mathrm{m}$ long tunable $\mathrm{PhC}$ directional coupler (Fig. 1(b), (c)). Parameters are similar to those in [10]: the waveguide width is $0.9 \sqrt{ } 3 \mathrm{a}$, radius $r=0.26 \mathrm{a}$, resulting into a beat length of $4 \mu \mathrm{m}$. An impedance matching section has been added to minimize reflection to less than $1 \%$. The whole coupler length is 20 periods $(\mathrm{a}=414 \mathrm{~nm}$ ).

The two arms of the interferometer are recombined by a $2 \times 2$ multimode interference (MMI) coupler. Inverted mode adapters have been added for efficient optical connection of the chip with lensed fibers, which drastically reduce reflections at the end facets $(<1 \%)$.

Tunability is achieved by a thermo-optic effect. The very small footprint of the $\mathrm{PhC}$ and the selective chemical etching of the underlying insulator allow a very efficient use of the heat generated by platinum micro-heaters deposited on both sides of the $\mathrm{PhC}$ (Fig. 1(c), (e)). As reported in [11], the $\mathrm{PhC}$ delay line is tunable over $70 \mathrm{ps}$ with a maximum of $80 \mathrm{~mW}$ of electrical power. The corresponding thermally induced phase shift is $1.9 \mathrm{rad} / \mathrm{mW}$. The full tuning range of the DC splitter is covered with $3.2 \mathrm{~mW}$ of applied electrical power [10]. The switching time constant is less than $2 \mu \mathrm{s}$.

\section{SMALl SignAl FREQUENCY RESPONSE}

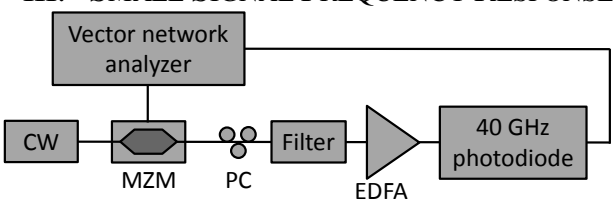

Fig. 2. Experimental set-up for characterization of the filter response

The small-signal frequency response of the filter was measured by externally modulating a continuous wave $(\mathrm{CW})$ optical carrier at $1550 \mathrm{~nm}$ with a swept single-frequency signal in $\mathrm{a} \mathrm{LiNbO}_{3}$ Mach-Zehnder modulator (MZM) and detecting the filtered signal in a 40-GHz photodiode (Fig. 2). An erbiumdoped fiber amplifier (EDFA) was placed after the filter to compensate its insertion losses (minimum $18 \mathrm{~dB}$ including coupling loss when arms are balanced). The $S_{21}$ scattering parameter was measured with a vector network analyzer. The normalized amplitude response of the filter when the signal is injected to port (1) and collected from port (3) of the chip (as

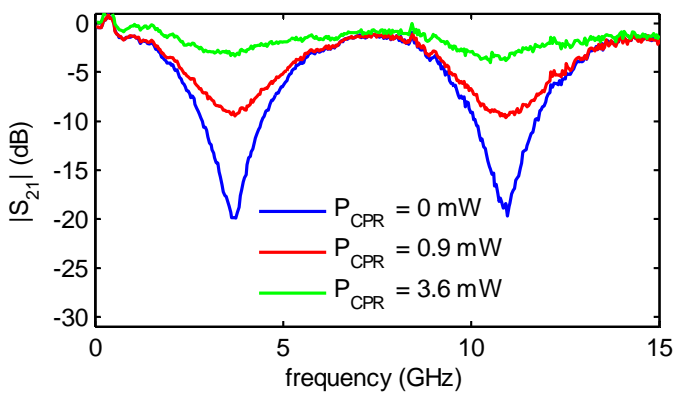

Fig. 3. Filter frequency responses when the $\mathrm{PhC}$ coupler is tuned. indicated in Fig. 1(a)) is represented in Fig. 3. Different filter weights were obtained by tuning the coupling ratio through the control of the electrical power $\left(\mathrm{P}_{\mathrm{CPR}}\right)$ applied to the $\mathrm{PhC}$ coupler. The free spectral range (FSR) of the filter, related to the propagation delay difference between the two arms, is also clearly visible. A maximum of $18 \mathrm{~dB}$ of signal rejection at the transmission dip is achieved when the optical power is suitably balanced between the two arms.

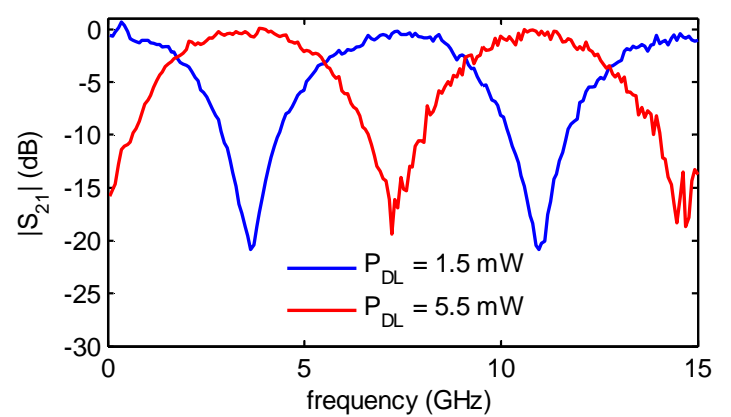

Fig. 4. Filter frequency responses when the $\mathrm{PhC}$ delay line is tuned.

As the $\mathrm{PhC}$ delay line is tuned by changing the control power $\left(\mathrm{P}_{\mathrm{DL}}\right)$ applied to the heater, the spectral response of the filter is changed accordingly, as shown in Fig. 4. Moreover, a fine- 
tuning of $\mathrm{P}_{\mathrm{DL}}$ allows control of the optical phase difference between the two arms, which ultimately enables changing the sign of the weighting coefficients. Due to the residual chirp of the external modulation, minima and maxima of the electric transmission spectrum can be exchanged (Fig. 4). The ability of having a minimum at zero frequency is a unique property of filters based on coherent summation. The $\pi$ phase shift required to switch the filter response between a maximum and a minimum at a given frequency results in a negligible variation of the propagation delay compared to the delay imbalance between the two arms.

\section{Device Functionality Assessment for Channel EQUALIZATION}

Chirp related to direct amplitude modulation of laser sources is a common issue in transmission links based on standard singlemode fibers (SSMFs), because of their large chromatic dispersion. The impairment can be mitigated using optical filtering [12], which improves the signal extinction ratio and flattens the channel response [13]. The device is assessed for this application with the setup shown in Fig. 5.

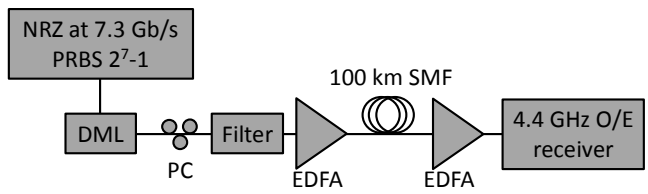

Fig. 5. Channel equalization experimental setup.

A $2^{7}-1$ pseudo-random binary sequence (PRBS) at $7.3 \mathrm{Gbit} / \mathrm{s}$ is applied to a directly-modulated laser (DML). The bias current is $65 \mathrm{~mA}$ and the peak-to-peak modulating voltage is $2 \mathrm{Vpp}$, resulting in an optical signal with $6 \mathrm{dBm}$ average power and $2.2 \mathrm{~dB}$ extinction ratio. The eye diagram of the signal at the output of the DML is shown in Fig. 6(a). The modulated signal is then coupled to the filter with its state-of-polarization adjusted to the TE mode of the waveguide thanks to a polarization controller (PC). The signal is then amplified by an EDFA before propagating through a $100-\mathrm{km}$ long SSMF and being detected in a $4.4-\mathrm{GHz}$ bandwidth opto-electronic $(\mathrm{O} / \mathrm{E})$ receiver. It is to notice that the filter could be placed indifferently before or after the fiber link.
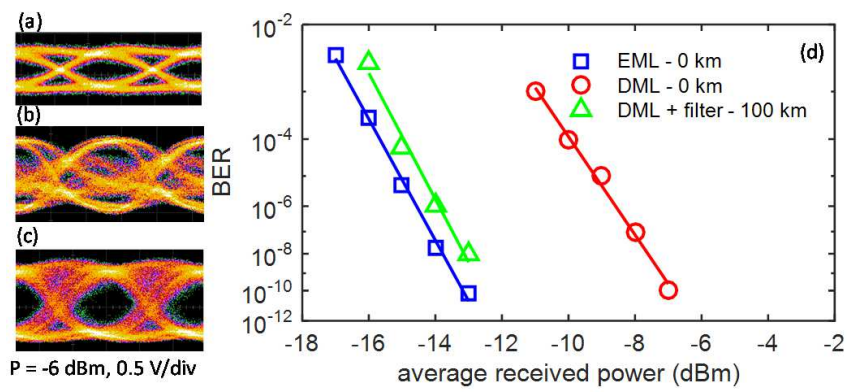

Fig. 6. Eye diagrams (a) at the output of the DML, (b) after $100 \mathrm{~km}$ without filter, and (c) with filter. (d) BER measurements.

As shown in Fig. 6(b), without filter, the eye diagram is strongly degraded after propagation due to the combination of chromatic dispersion and chirp of the DML. After introducing the filter and adjusting the depth and frequency position of the dips in its transfer function, the opening of the eye diagram is significantly improved at the receiver side (Fig. 6(c)). In this experiment, best operation was obtained with $0.8 \mathrm{~mW}$ and $3 \mathrm{~mW}$ electrical powers applied to the directional coupler and to the delay line sections, respectively. For a quantitative assessment, the bit-error-rate (BER) is measured in the "backto-back" configuration (transmitter directly connected to the receiver, without the filter) for the signal generated in the DML, and compared to the case where an externally modulated laser (EML) is used (still without filter). The corresponding BER curves in Fig. 6(d) reveal a power penalty related to the limited extinction ratio of the DML. After propagation over $100 \mathrm{~km}$ of SSMF of the directly modulated signal without any filter, the BER cannot even be measured. Introducing the filter after the DML leads to a substantial improvement of the BER, which is now comparable to the reference case (EML source), but after $100 \mathrm{~km}$ propagation. The residual penalty compared to this reference case is of the order of $1 \mathrm{~dB}$.

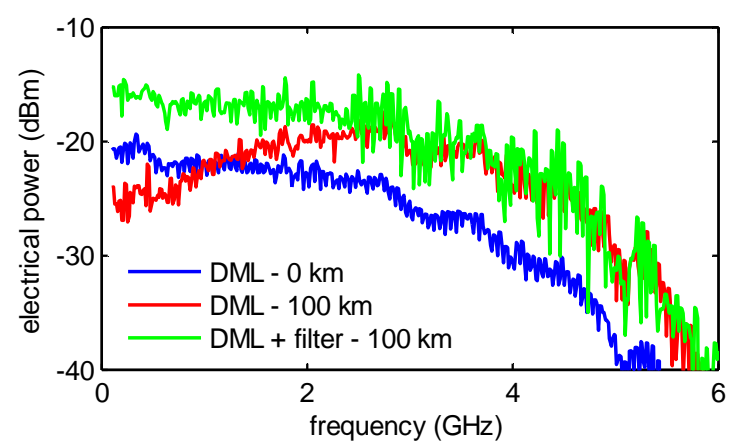

Fig. 7. RF spectra of the detected signal at the DML output and after transmission over $100 \mathrm{~km} \mathrm{SSMF}$ without and with filter.

In order to visualize the impact of the filter on the frequency response of the channel (DML with or without filter + SSMF + photodiode) the electrical spectra of the received signals are shown in Fig. 7. Propagation over $100 \mathrm{~km}$ of SSMF induces an 8 -dB dip in the low frequency part of the spectrum compared to the back-to-back case. The origin of this dip is the interaction between fiber dispersion and DML frequency chirp [14]. This dip is removed when the filter is used after the DML due to the low pass operation of the filter which favors low frequencies. The photonic RF filter consequently equalizes the channel response and leads to a better tolerance to chromatic dispersion of the DML link. In the system experiment, low frequencies in the modulated signal, which were cut in the case without filter are now properly transmitted, which leads to improved BER performance.

We can also notice a shift of the RF spectrum of the filtered signal after $100 \mathrm{~km}$ propagation towards higher power compared to the reference without propagation, despite the mean optical power on the photodiode being the same in both cases. Indeed the filter, in addition to RF low pass filtering, also plays a role in the optical domain. Direct carrier modulation of the laser leads to different instantaneous frequencies for symbols ' 1 ' and ' 0 ' as a result of adiabatic chirp [15], as can be seen in the optical spectrum of Fig. 8 measured using an optical spectrum analyzer with $160 \mathrm{fm}$ resolution. The shorter wavelength peak corresponds to symbols ' 1 ' being transmitted, while the longer wavelength peak corresponds to symbols ' 0 '. 


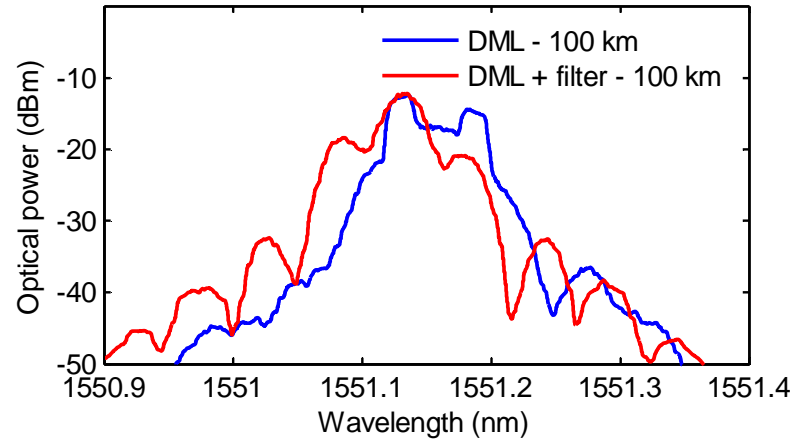

Fig. 8. Optical spectra of the signal after transmission over $100 \mathrm{~km} \mathrm{SSMF}$ without and with filter.

The optical RF filter is slightly shifted from the central frequency of the directly modulated spectrum in order to favor symbols ' 1 ' and attenuate symbols ' 0 ' (Fig. 8, spectrum measured with filter). The attenuation of symbols ' 0 ' through the filter improves the signal extinction ratio, which in turn improves the BER performance in term of sensitivity. The resulting higher modulation index finally leads to enhanced RF power after detection as depicted in Fig. 7.

\section{CONCLUSION}

A high-performance and compact SOI 2-tap interferometer used as a channel equalizer for transmission of directly modulated signals over a dispersive fiber link has been demonstrated. The transfer function of the photonic RF filter is fully adjustable in amplitude and frequency thanks to thermally-tunable $\mathrm{PhC}$ couplers and delay lines. The compactness and the dispersive nature of $\mathrm{PhCs}$ enable powerefficient operation, with electric driving power in the $\mathrm{mW}$ range. This enabled channel fading compensation of a $100-\mathrm{km}$ long link supporting a directly modulated telecommunication signal, leading to a clear eye diagram opening and the possibility to recover the bit-error-rate of a reference signal with less than 1-dB power penalty. This experiment demonstrates the maturity of the technology in particular for $\mathrm{PhC}$ based elements. Their realization was done in a standard research clean room environment using ebeam lithography. However the process is fully compatible with CMOS process as has been demonstrated by many groups on different foundries [16] -[20]. It opens the way to photonic RF filters concatenating several levels of this elementary cell in order to reach higher number of tap levels and more flexible filtering functions. Furthermore this technology can also be suitable for applications such as arbitrary waveform generation [21], pulse shaping [22] or beam forming [23].

\section{REFERENCES}

[1] J. Capmany, B. Ortega, and D. Pastor, "A tutorial on microwave photonic filters,” J. Lightw. Technol., vol. 24, no. 1, pp. 201-229, Jan. 2006.

[2] J. Capmany, B. Ortega, D. Pastor, and S. Sales, "Discrete-time optical processing of microwave signals," J. Lightw. Technol., vol. 23, no. 2, pp. 702-722, Feb. 2005.

[3] F. Coppinger, S. Yegnanarayanan, P.D. Trinh, and B. Jalali, “All-optical incoherent negative taps for photonic signal processing”, Electron. Lett., vol. 33, no. 11, pp. 973-975, May 1997.

[4] J. Capmany, D. Pastor, A. Martinez, B. Ortega, and S. Sales, "Microwave photonic filters with negative coefficients based on phase inversion in an electro-optic modulator", Opt. Lett., vol. 28, no. 16, pp. 1415-1417, Aug. 2003
[5] M. Sagues, A. Loayssa, and J. Capmany, "Multitap complex coefficient incoherent microwave photonic filters based on stimulated Brillouin scattering", IEEE Photon. Technol. Lett, vol. 19, no.16, pp. 1194-1196, Aug. 2007.

[6] E. J. Norberg, R. S. Guzzon, J. S. Parker, L. A. Johansson, and L. A. Coldren, "Programmable photonic microwave filters monolithically integrated in InP/InGaAsP," J. Lightw. Technol., vol. 29, no. 11, pp. 1611-1619, Jun. 2011.

[7] N. N. Feng, P. Dong, D. Feng,W. Qian, H. Liang, D. C. Lee, J. B. Luff, A. Agarwal, T. Banwell, R. Menendez, P. Toliver, T. K. Woodward, and M. Asghari, "Thermally-efficient reconfigurable narrowband RFphotonic filter," Opt. Express, vol. 18, pp. 24648-24653, Nov. 2010.

[8] J. Sancho, J. Bourderionnet, J. Lloret, S. Combrié, I. Gasulla, S. Xavier, S. Sales, P. Colman, G. Lehoucq, D. Dolfi, J. Capmany and A. De Rossi "Integrable microwave filter based on a photonic crystal delay line" Nature Communications, vol. 3, article 1075, Sep. 2012.

[9] Y. Hamachi, S. Kubo, and T. Baba, "Slow light with low dispersion and nonlinear enhancement in a lattice-shifted photonic crystal waveguide," Opt. Lett. 34, 1072-1074 (2009).

[10] Z. Han, G. Moille, X. Chécoury, J. Bourderionnet, G. Lehoucq, P. Boucaud, A. De Rossi and S. Combrié, "High-performance and powerefficient $2 \times 2$ optical switch on silicon-on-insulator," Opt. Express, vol. 23, no. 19, pp. 24163-24170, Sep. 2015.

[11] J. Bourderionnet, S. Combrié, Z. Han, X. Chécoury, P. Boucaud, and A. De Rossi, "Photonic crystal SOI-PIC for integrated microwave photonics filters," European Conference on Lasers and Electro-Optics, Germany, 2015, paper CK7.3.

[12] D. Mahgerefteh, Y. Matsui, X. Zheng, and K. McCallion, "Chirp managed laser and applications," IEEE J. Sel. Topics Quantum Electron., vol. 16, no. 5, pp. 1126-1139, Mar. 2010.

[13] M. McAdams, E. Peral, D. Provenzano, W. K. Marshall and A. Yariv, "Improved laser modulation response by frequency modulation to amplitude modulation conversion in transmission through a fiber grating", Appl. Phys. Lett., vol. 71, no. 7, pp. 879-881, 1997.

[14] B. Wedding, "Analysis of fibre transfer function and determination of receiver frequency response for dispersion supported transmission," in Electron. Lett. , vol.30, no.1, pp.58-59, 6 Jan. 1994.

[15] J. Yu, Z. Jia, M.-F. Huang, M. Haris, P. N. Ji, T Wang and G.-K. Chang "Applications of $40-\mathrm{Gb} / \mathrm{s}$ chirp-managed laser in access and metro networks", J. Lightw. Technol., vol. 27, no.3, pp. 253-265, Feb. 2009.

[16] M. Settle, M. Salib, A. Michaeli, and Thomas F. Krauss. "Low loss silicon on insulator photonic crystal waveguides made by $193 \mathrm{~nm}$ optical lithography" Optics Express, vol. 14, no. 6, pp 2440-2445, Mar. 2006.

[17] S.K. Selvaraja, P. Jaenen, W. Bogaerts, D. Van Thourhout, P. Dumon, and R. Baets. "Fabrication of photonic wire and crystal circuits in siliconon-insulator using 193-nm optical lithography" J. Lightw. Technol., vol. 27, no. 18, pp. 4076-4083, Jan. 2009.

[18] N. Ishikura, R. Hosoi, R. Hayakawa, T. Tamanuki, M. Shinkawa, and T. Baba. "Photonic crystal tunable slow light device integrated with multiheaters", Appl. Phys. Lett., vol. 100, no. 22, article 221110, May 2012.

[19] C. V. Poulton, X. Zeng, M. T. Wade, J. M. Shainline, J. S. Orcutt, and M. A. Popović "Photonic crystal microcavities in a microelectronics 45-nm SOI CMOS technology” IEEE Photon. Technol. Lett., vol. 27, no. 6, pp. 665-668, Mar. 2015.

[20] Y. Ooka, T. Tetsumoto, A. Fushimi, W. Yoshiki, and T. Tanabe "CMOS compatible high-Q photonic crystal nanocavity fabricated with photolithography on silicon photonic platform" Scientific Reports, vol. 5, article 11312, Jun. 2015.

[21] S. Liao, Y. Ding, J. Dong, T. Yang, X. Chen, D. Gao, and X. Zhang "Arbitrary waveform generator and differentiator employing an integrated optical pulse shaper." Opt. Express, vol. 23, no. 9, pp. 12161-12173, Apr. 2015.

[22] H. Bazargani, M. Burla, and J. Azaña, "Experimental demonstration of sub-picosecond optical pulse shaping in silicon based on discrete spaceto-time mapping," Opt. Lett., vol. 40, no. 23, pp. 5423-5426, 2015.

[23] Z. Cao, Q. Ma, A. B. Smolders, Y. Jiao, M. J. Wale, C. W. Oh, H. Wu, and A. M. J. Koonen, "Advanced integration techniques on broadband millimeter-wave beam steering for $5 \mathrm{G}$ wireless networks and beyond," IEEE J. Quantum Electron., vol. 52, no. 1, pp. 1-20, Jan. 2016. 